International Journal of Pure and Applied Mathematics

Volume 85 No. 5 2013, 965-981

ISSN: 1311-8080 (printed version); ISSN: 1314-3395 (on-line version)

url: http://www.ijpam.eu

doi: http://dx.doi.org/10.12732/ijpam.v85i5.14

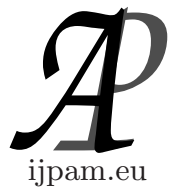

\title{
ON COMPARISONS OF CHEBYSHEV-HALLEY ITERATION FUNCTIONS BASED ON THEIR ASYMPTOTIC CONSTANTS
}

\author{
F. Dubeau \\ Department of Mathematics \\ University of Sherbrooke \\ 2500 University Boul., Sherbrooke (Qc), CANADA, J1K 2R1
}

\begin{abstract}
Using the Chebyshev-Halley family of iteration functions and numerical examples, we discuss the influence of both the asymptotic constant and the choice of the initial trial in the basin of attraction on the rapidity of convergence of an iterative method.
\end{abstract}

AMS Subject Classification: 11B37, 65B99, 65D99, 65H05

Key Words: Chebyshev-Halley iteration functions, asymptotic constant, order of convergence, approximate asymptotic constant, local order of convergence

\section{Introduction}

Methods for solving a nonlinear equation are classified by their order of convergence $p$ and the number $d$ of function (and derivatives) evaluations per step. Based on $p$ and $d$, there are two efficiency measures defined by $I=p / d$ (informational efficiency) and $E=p^{1 / d}$ (efficiency index) [12]. These measures do not depend on the function $f(x)$ nor on the number of steps required to solve the problem within a given precision. Unfortunately, for methods of the same order $p$ and demanding the same number of function evaluations $d$, these two measures are the same for these methods. For example, each iteration function (IF) of the Chebyshev-Halley family is of order $p=3$ and requires $d=3$ evaluations per step (evaluation of $f(x), f^{(1)}(x)$, and $f^{(2)}(x)$ ).

Received: May 29, 2013

(c) 2013 Academic Publications, Ltd. url: www.acadpubl.eu 
Another measure introduced recently is the basin of attraction of a given IF ([10] and the references therein). The basin of attraction depends on $f(x)$ and, for a given method of order $p$, the "local order" of convergence in the basin is not necessarily $p$ for the first steps of the method.

Moreover, since the number of steps required to reach a given precision is not known, it is difficult to classify methods with respect to the efficiency index, the informational efficiency, and even their basins of attraction (which depend on $f(x))$.

In many papers, to compare methods authors take one arbitrary initial point and study the error after the same number of steps or the number of steps required to get a given error bounds (see for examples [2, 11]). This number of step depends on how far the initial trial $x_{0}$ is from the solution $\alpha$, and the value of the asymptotic constant. For two methods of the same order $p$, the method having the smallest asymptotic constant will converge faster than the second method having the higher asymtotic constant, for a starting point $x_{0}$ sufficiently close to the solution $\alpha$. Unfortunately, as for the basin of attraction, the asymtotic constant depends on the function $f(x)$.

One goal of this paper is to illustrate the role of the asymptotic constants in the rapidity of convergence of iterative methods and show that it is difficult to classify IFs based on the asymptotic constant and the basin of attraction.

In Section 2 we introduce definitions and basic results on asymptotic constants and order of convergence. In Section 3 we present a one parameter formula valid to describe each member of the Chebyshev-Halley family of IFs of order 3, and an expression for their asymptotic constants. In Section 4, to illustrate the points raised in the introduction about te comparison of IFs, we present numerical experiments using the Chebyshev-Halley family of IFs of order 3. At first, we apply the IFs on set of 5 test functions already used in [11] to compare similar IFs. We choose initial points $x_{0}$ in the basin of attraction of the considered solution $\alpha$ and study the role of the asymptotic constant on the convergence speed. In second, we consider a form of particular test functions for which IFs of the Chebyshev-Halley family are of order $p$ for arbitrary $p \geq 3$. Finally we consider the $n$-th root computation problem of a real number to compare the asymptotic constant of the methods. Concluding remarks are given in Section 5. 


\section{Preliminaries}

In this section we assume that $\alpha$ is a fixed point of an iteration function (IF) $G(x)$ which is sufficiently many times differentiable in a neighborhood of $\alpha$. Moreover we consider any sequence $\left\{x_{k+1}=G\left(x_{k}\right)\right\}_{k=0}^{+\infty}$ starting at an arbitrary point $x_{0}$ and converging to $\alpha$ ( $x_{0}$ is in the basin of attraction of $\alpha$ with respect to $G(x))$.

Let $e_{k}=x_{k}-\alpha$ be the error at the step $k$. We say that $p$ (a positive integer) is the order of convergence of the sequence $\left\{x_{k+1}\right\}_{k=0}^{+\infty}$ to $\alpha$ if and only if

$$
\lim _{k \rightarrow+\infty} \frac{e_{k+1}}{e_{k}^{p}}=K_{p}(\alpha ; G) \in \mathbb{R} \backslash\{0\} .
$$

We also say that $G(x)$ in an IF of ordre $p . K_{p}(\alpha ; G)$ is called the asymptotic constant (for the order $p$ ). For $p=1$ we assume that $\left|K_{1}(\alpha ; G)\right|<1$. If the limit $K_{p}(\alpha ; G)$ exists but is zero, we can say that $G(x)$ is of ordre at least $p$.

Let us observe that if $K_{p}(\alpha ; G)$ exists then for any integer $p^{\prime}$, such that $1 \leq p^{\prime}<p$, we have $K_{p^{\prime}}(\alpha ; G)=0$. Indeed, since $p-p^{\prime}>0$, we can write

$$
\frac{e_{k+1}}{e_{k}^{p^{\prime}}}=\frac{e_{k+1}}{e_{k}^{p}} e_{k}^{p-p^{\prime}},
$$

and the result follows by taking the limit.

Since we have assumed that $G(x)$ is sufficiently many times differentiable, we have

$$
K_{p}(\alpha ; G)=\frac{G^{(p)}(\alpha)}{p !} .
$$

In fact the limit $K_{p}(\alpha ; G)$ exists if and only if $G(\alpha)=\alpha, G^{(l)}(\alpha)=0$ for $l=1, \ldots, p-1$, and $G^{(p)}(\alpha) \neq 0[12]$.

From a numerical point of view, since $\alpha$ is not known, let $\tilde{e}_{k}=x_{k}-x_{k+1}$ be the approximate error at the step $k$. It is useful to define the ratio

$$
\widetilde{K}_{p}(\alpha ; k)=\frac{\tilde{e}_{k+1}}{\tilde{e}_{k}^{p}},
$$

which can be called the approximate asymptotic constant (of order $p$ ) at the point $x_{k}$. Since

$$
\widetilde{K}_{p}(\alpha ; k)=\frac{e_{k+1}}{e_{k}^{p}} \cdot \frac{1-\frac{e_{k+2}}{e_{k+1}}}{\left[1-\frac{e_{k+1}}{e_{k}}\right]^{p}},
$$


it follows that for $p=1$

$$
\lim _{k \rightarrow+\infty} \widetilde{K}_{1}(\alpha ; k)=K_{1}(\alpha ; G) \cdot \frac{1-K_{1}(\alpha ; G)}{1-K_{1}(\alpha ; G)}=K_{1}(\alpha ; G)
$$

since $K_{1}(\alpha ; G) \neq 1$, and for $p>1$

$$
\lim _{k \rightarrow+\infty} \widetilde{K}_{p}(\alpha ; k)=K_{p}(\alpha ; G) \cdot \frac{1-K_{1}(\alpha ; G)}{\left[1-K_{1}(\alpha ; G)\right]^{p}}=K_{p}(\alpha ; G)
$$

because $K_{1}(\alpha ; G)=0$.

In the same way, to estimate the order $p$ of convergence, we can proceed as follows. Since

$$
\begin{aligned}
\left|\widetilde{K}_{1}(\alpha ; k+1)\right| & =\left|\frac{\tilde{e}_{k+2}}{\tilde{e}_{k+1}}\right| \\
& =\frac{\left|\widetilde{K}_{p}(\alpha ; k+1)\right|}{\left|\widetilde{K}_{p}(\alpha ; k)\right|}\left|\frac{\tilde{e}_{k+1}}{\tilde{e}_{k}}\right|^{p} \\
& =\frac{\left|\widetilde{K}_{p}(\alpha ; k+1)\right|}{\left|\widetilde{K}_{p}(\alpha ; k)\right|}\left|\widetilde{K}_{1}(\alpha ; k)\right|^{p},
\end{aligned}
$$

it follows that

$$
p=\frac{\ln \left|\widetilde{K}_{1}(\alpha ; k+1)\right|}{\ln \left|\widetilde{K}_{1}(\alpha ; k)\right|}-\frac{\ln \left|\frac{\widetilde{K}_{p}(\alpha ; k+1)}{\widetilde{K}_{p}(\alpha ; k)}\right|}{\ln \left|\widetilde{K}_{1}(\alpha ; k)\right|} .
$$

Because

$$
\lim _{k \rightarrow+\infty} \ln \left|\frac{\widetilde{K}_{p}(\alpha ; k+1)}{\widetilde{K}_{p}(\alpha ; k)}\right|=0
$$

and

$$
\lim _{k \rightarrow+\infty} \ln \left|\widetilde{K}_{1}(\alpha ; k)\right|=-\infty,
$$

we can define the local order of convergence at $x_{k}$ by

$$
\tilde{p}\left(x_{k}\right)=\frac{\ln \left|\widetilde{K}_{1}(\alpha ; k+1)\right|}{\ln \left|\widetilde{K}_{1}(\alpha ; k)\right|},
$$

and the order $p$ can be obtained by considering the limit

$$
p=\lim _{k \rightarrow+\infty} \tilde{p}\left(x_{k}\right) \text {. }
$$




\section{Chebyshev-Halley Family of Iteration Functions}

The basic problem is to look for $\alpha \in \mathbb{R}$ such that $f(\alpha)=0$. We assume that $f^{(1)}(\alpha) \neq 0$ and $\alpha$ is a simple zero of $f(x)$. In this section we recall expressions for the IF and its asymptotic constant valid for each members of the ChebyshevHalley family of IFs of order 3 .

Following [4], let $N_{\beta}(x)$ be the Newton's IF of order 2 applied to $f(x) /\left[f^{(1)}\right.$ $(x)]^{\beta}$ with $\beta \in \mathbb{R}$. Then using $\beta \neq 0$, we set

$$
\begin{aligned}
G_{\beta}(x) & =\frac{1}{2 \beta}\left[N_{\beta}(x)-(1-2 \beta) N_{0}(x)\right] \\
& =x-\frac{f(x)}{f^{(1)}(x)}\left[\frac{1-(\beta-1 / 2) L_{f}(x)}{1-\beta L_{f}(x)}\right]
\end{aligned}
$$

where $L_{f}(x)=\frac{f(x) f^{(2)}(x)}{\left[f^{(1)}(x)\right]^{2}}$. This one parameter family of IFs corresponds to the Chebyshev-Halley family of IFs of order 3 (see also $[3,13,1,7,8]$ ). Indeed, for $\beta=1$ we get the Super-Halley IF, for $\beta=1 / 2$ we get the Halley IF, and for $\beta=0$, which is a limit case, we get the Chebyshev IF. We verify that

$$
G_{\beta}(\alpha)=\alpha, \quad G_{\beta}^{(1)}(\alpha)=0, \quad G_{\beta}^{(2)}(\alpha)=0,
$$

and

$$
G_{\beta}^{(3)}(\alpha)=-\frac{f^{(3)}(\alpha)}{f^{(1)}(\alpha)}+3(1-\beta)\left[\frac{f^{(2)}(\alpha)}{f^{(1)}(\alpha)}\right]^{2} .
$$

Then for any $\beta$ we have an IF of order 3 with an asymptotic constant

$$
K_{3}\left(\alpha ; G_{\beta}\right)=\frac{1}{3 !}\left[-\frac{f^{(3)}(\alpha)}{f^{(1)}(\alpha)}+3(1-\beta)\left[\frac{f^{(2)}(\alpha)}{f^{(1)}(\alpha)}\right]^{2}\right]^{.}
$$

\section{Numerical Examples}

To illustrate the points raised in the introduction about te comparison of IFs, we present numerical experiments using the Chebyshev-Halley family of IFs of order 3. We consider 3 types of examples to illustrate different phenomenas that might happen when we try to compare IFs. At first, we apply the IFs on set of 5 test functions already used in [11] to compare similar IFs. We choose initial points $x_{0}$ in the basin of attraction of the considered solution $\alpha$ and 
study the role of the asymptotic constant on the convergence speed. In second, we consider a form of particular test functions for which IFs of the ChebyshevHalley family are of order $p$ for arbitrary $p \geq 3$. Finally we consider the $n$-th root computation problem of a real number and compare the asymptotic constant of the methods. In this section we use the notation $E_{k}=\left|e_{k}\right|$ and $\widetilde{E}_{k}=\left|\tilde{e}_{k}\right|$.

\subsection{Test Functions}

The 5 test functions, already used in [11] to compare some IFs, are given explicitely in Table 1 with the root $\alpha$ we are interested in. To illustrate that the asymptotic constant depends on the function $f(x)$, Table 2 presents the asymptotic constants $K_{3}\left(\alpha, G_{\beta}\right)$, for $\beta=0,1 / 2$, and 1 , when they are applied to the 5 test functions.

In Tables 3 and 4, we report standard normal situations. If we use an $x_{0}$ near enough to $\alpha$ the method with the smallest asymptotic constant will converge faster.

In Table 5, we took two initial points $x_{0}$, the first value near the exact solution $\alpha$ and the second value relatively far from the solution $\alpha$ but both in the basin of attraction of $\alpha$ with respect to $G_{\beta}$. For the first initial $x_{0}, G_{0}$ converge faster than the two other methods, but for the second initial point $x_{0}$, it is $G_{1 / 2}$ which seems to converge faster (after 5 iterations) even if its asymptotic constant is not the smallest one.

In Table 6, taking different initial points, the estimated error $\widetilde{E}_{5}$ is given for each method. It illustrates the fact that the error decreasing depends on the initial point $x_{0}$ in the basin of attraction of the method $G_{\beta}$. Looking for $\alpha=-3$, depending on how far from the root $\alpha$ we start the process, a method can show faster converge than another method even if its asymptotic constant is higher than the other for the problem considered. This is the case for $G_{1 / 2}$ compared to $G_{1}$ for $x_{0}=-2.2,-2.4$, and -2.6 .

In Table 7, we have an example of an initial point which show faster convergence for $G_{0}$ diring the first 3 iterations, but after that it is $G_{1 / 2}$ which shows the best error $\widetilde{E}_{k}$. Let us use the notation $E^{\beta, k}$ for $E_{k}$ obtained from $G_{\beta}$. If we suppose that $E_{\beta, k+1} \approx\left|K_{3}\left(\alpha, G_{\beta}\right)\right| E_{\beta, k}^{p}$, using approximate value we can deduce that

$$
\frac{E_{\beta, k+1}}{E_{\beta^{\prime}, k+1}} \approx\left|\frac{K_{3}\left(\alpha, G_{\beta}\right)}{K_{3}\left(\alpha, G_{\beta^{\prime}}\right)}\right|^{\frac{p^{k}-1}{p-1}}\left[\frac{E_{\beta, 1}}{E_{\beta^{\prime}, 1}}\right]^{p^{k}}
$$




\begin{tabular}{|l|r|}
\hline \multicolumn{1}{|c|}{ Function $f(x)$} & \multicolumn{1}{c|}{$\alpha$} \\
& \\
\hline \hline$f_{1}(x)=x-2-e^{-x}$ & $2.1200282389 \ldots$ \\
\hline$f_{2}(x)=e^{x}-3 x^{2}$ & $-0.4589622675 \ldots$ \\
\hline$f_{3}(x)=x^{2}+(x-1)^{5}$ & $0.3459548158 \ldots$ \\
\hline$f_{4}(x)=x^{3}+5 x^{2}+10 x+12$ & $-3.0000000000 \ldots$ \\
\hline$f_{5}(x)=x-(x-1)^{20}$ & $0.1061045880 \ldots$ \\
\hline
\end{tabular}

Table 1: Set of the 5 test functions used to compare the methods.

$$
=\left|\frac{K_{3}\left(\alpha, G_{\beta}\right)}{K_{3}\left(\alpha, G_{\beta^{\prime}}\right)}\right|^{-\frac{1}{p-1}}\left[\left|\frac{K_{3}\left(\alpha, G_{\beta}\right)}{K_{3}\left(\alpha, G_{\beta^{\prime}}\right)}\right|^{\frac{1}{p-1}}\left[\frac{E_{\beta, 1}}{E_{\beta^{\prime}, 1}}\right]\right]^{p^{k}} .
$$

In our case $p=3$, and taking $\beta=1 / 2$ and $\beta^{\prime}=0$, we have

$$
\left|\frac{K_{3}\left(\alpha, G_{1 / 2}\right)}{K_{3}\left(\alpha, G_{0}\right)}\right|^{1 / 2}=\left(\frac{5.725}{61.64}\right)^{1 / 2} \approx 0.3048
$$

and

$$
\frac{E_{1 / 2,1}}{E_{0,1}} \approx \frac{1.14 \cdot 10^{-2}}{4.45 \cdot 10^{-3}} \approx 2.562
$$

It follows that

$$
\frac{E_{1 / 2, k+1}}{E_{0, k+1}} \approx \frac{0.7807^{3^{k}}}{.3048}
$$

which indicates that after few iterations, even if $E_{1 / 2,1}>E_{0,1}$ we will have $E_{1 / 2, k+1}<E_{0, k+1}$ after $k$ iterations. In Table $7, E_{1 / 2,1}>E_{0,1}$ but $E_{1 / 2,4}<E_{0,4}$.

\subsection{A Special Family of Test Functions}

The following function is also used for comparison in [11]

$$
F(x)=(x-1)-(x-1)^{20} .
$$

It is a member of a family of functions of the form

$$
F_{p}(x)=a_{1}(x-\alpha)+a_{p}(x-\alpha)^{p}+a_{p+1}(x)(x-\alpha)^{p+1},
$$




\begin{tabular}{|c||c|c|c|}
\cline { 2 - 4 } \multicolumn{1}{c||}{} & \multicolumn{3}{c|}{ Asymptotic constant $K_{3}\left(\alpha, G_{\beta}\right)$} \\
\cline { 2 - 4 } & Chebyshev & Halley & Super-Halley \\
\hline & & & \\
\hline & & & $\beta=1$ \\
\hline & & & \\
\hline$f_{1}(x)$ & $\mathbf{- 0 . 0 1 2 1 2}$ & -0.01499 & -0.01786 \\
\hline$f_{2}(x)$ & 1.226 & 0.5974 & $\mathbf{- 0 . 0 3 1 1 0}$ \\
\hline$f_{3}(x)$ & $\mathbf{- 0 . 1 5 9}$ & -1.410 & $\mathbf{- 2 . 6 6 3}$ \\
\hline$f_{4}(x)$ & 0.5102 & 0.1837 & $\mathbf{- 0 . 1 4 2 9}$ \\
\hline$f_{5}(x)$ & 61.64 & $\mathbf{5 . 7 2 5}$ & -50.19 \\
\hline
\end{tabular}

Table 2: Asymptotic constants for the Chebychev, Halley and SuperHalley IFs for the 5 test functions (the smallest asymptotic constant are indicated in boldface).

where $p \geq 3, a_{1}$ and $a_{p}$ are two nonzero constants, and $a_{p+1}(x)$ is a regular function.

These functions are such that $F_{p}(\alpha)=0, F_{p}^{(1)}(\alpha)=a_{1} \neq 0, F_{p}^{(j)}(\alpha)=0$ for $j=2, \ldots, p-1$, and $F_{p}^{(p)}(\alpha) / p !=a_{p} \neq 0$. It is well known that for that type of functions, the Newton's method is of order $p$ for any $p \geq 2[6]$. Since $F_{p}(x) /\left[F_{p}^{(1)}(x)\right]^{\beta}$ is of the same type, the Newton's method applied to $F_{p}(x) /\left[F_{p}^{(1)}(x)\right]^{\beta}$ will be of the same order $p$. It follows that $G_{\beta}$ will be also of order $p$ for any $p \geq 3$ independently of the value of $\beta$. We can also show that

$$
K_{p}\left(\alpha, G_{\beta}\right)=-a_{p} \frac{(p-1)(p-2)}{2}
$$

In Table 8, we illustrate the convergence of the IFs when applied to $F_{p}(x)$ for $p=3, p=5$, and $p=20$, with $a_{1}=1, a_{p}=-1$ and $a_{p+1}=0$. Their given asymptotic constants are estimated and correspond to the values obtained using (14). 


\begin{tabular}{|c|c|c|c|}
\hline \multirow[t]{2}{*}{$f_{1}(x)$} & \multicolumn{3}{|c|}{ Absolute error $\widetilde{E}_{5}$ for $k=1, \ldots, 5$} \\
\hline & Chebyshev & Halley & Super-Halley \\
\hline \multirow[t]{2}{*}{$k$} & $\beta=0$ & $\beta=1 / 2$ & $\beta=1$ \\
\hline & \multicolumn{3}{|c|}{$\overline{x_{0}=2.1 \text { and } \alpha=2.1200282389 \ldots}$} \\
\hline 1 & $9.79 \cdot 10^{-8}$ & $1.22 \cdot 10^{-7}$ & $1.46 \cdot 10^{-7}$ \\
\hline 2 & $1.14 \cdot 10^{-23}$ & $2.70 \cdot 10^{-23}$ & $5.52 \cdot 10^{-23}$ \\
\hline 3 & $1.78 \cdot 10^{-71}$ & $2.96 \cdot 10^{-70}$ & $3.00 \cdot 10^{-69}$ \\
\hline 4 & $6.83 \cdot 10^{-215}$ & $3.91 \cdot 10^{-211}$ & $4.83 \cdot 10^{-208}$ \\
\hline 5 & $3.85 \cdot 10^{-645}$ & $8.94 \cdot 10^{-634}$ & $2.01 \cdot 10^{-624}$ \\
\hline 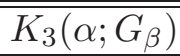 & -0.01212 & -0.01499 & -0.01786 \\
\hline
\end{tabular}

Table 3: Error decreasing and asymptotic constants (best errors and the smallest asymptotic constant are indicated in boldface).

\subsection{The $n$-th Root Computation Problem}

The problem of finding the $n$-th root $\alpha=r^{1 / n}$ of a positive real number $r$ is equivalent to solve the equation $f(x)=x^{n}-r=0$. Since $f^{(1)}(x)=n x^{n-1}$, $f^{(2)}(x)=n(n-1) x^{n-2}$, and $f^{(3)}(x)=n(n-1)(n-2) x^{n-3}$, it follows that

$$
K_{3}\left(\alpha ; G_{\beta}\right)=\frac{(n-1)^{2}}{2 \alpha^{2}} C(\beta)
$$

where

$$
C(\beta)=\frac{2 n-1}{3(n-1)}-\beta .
$$

It follows that for

$$
\beta^{*}(n)=\frac{2 n-1}{3(n-1)},
$$

which does not depend on $\alpha$ but only on $n, C\left(\beta^{*}(n)\right)=0$ and $K_{3}\left(\alpha ; G_{\beta^{*}(n)}\right)=0$ and the method is of order 4 (see also [9]). 


\begin{tabular}{|c|c|c|c|}
\hline \multirow[t]{2}{*}{$f_{2}(x)$} & \multicolumn{3}{|c|}{ Absolute error $\widetilde{E}_{5}$ for $k=1, \ldots, 5$} \\
\hline & Chebyshev & Halley & Super-Halley \\
\hline \multirow[t]{2}{*}{$k$} & $\beta=0$ & $\beta=1 / 2$ & $\beta=1$ \\
\hline & \multicolumn{3}{|c|}{$x_{0}=-0.5$ and $\alpha=-0.4589622675 \ldots$} \\
\hline 1 & $7.40 \cdot 10^{-05}$ & $3.77 \cdot 10^{-05}$ & $8.24 \cdot 10^{-07}$ \\
\hline 2 & $4.97 \cdot 10^{-13}$ & $3.21 \cdot 10^{-14}$ & $1.74 \cdot 10^{-20}$ \\
\hline 3 & $1.50 \cdot 10^{-37}$ & $1.98 \cdot 10^{-41}$ & $1.64 \cdot 10^{-61}$ \\
\hline 4 & $4.18 \cdot 10^{-111}$ & $4.63 \cdot 10^{-123}$ & $1.38 \cdot 10^{-184}$ \\
\hline 5 & $8.96 \cdot 10^{-332}$ & $5.96 \cdot 10^{-368}$ & $8.19 \cdot 10^{-554}$ \\
\hline 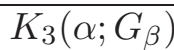 & 1.226 & 0.5974 & -0.03110 \\
\hline
\end{tabular}

Table 4: Error decreasing and asymptotic constants (best errors and the smallest asymptotic constant are indicated in boldface).

In Table 9 we have compared values of $C(\beta)$ for the 3 popular IFs applied to this particular problem. We observe that for low values of $n(2 \leq n \leq 4)$ the Super-Halley IF has the smallest asymptotic constant, less than the Halley IF. For values of $n \geq 6$ the Halley IF has the lowest asymptotic constant, and for $n=5$ they are equivalent. The Chebyshev IF always has a greater asymtotic constant compared to the two other IFs.

\section{Conclusion}

From the numerical examples given here we cannot say that a method is superior to the other methods among the Chebyshev, Halley or Super-Halley methods. As observed, it is not easy to compare IFs of the same order of convergence $p$ and requiring the same number of function evaluations $d$. For methods of the same order of convergence, the method with the smallest asymptotic constant will be locally faster, but if the first trial is too far from the solution we are looking for, it might happend that a method with a higher asymptotic constant 


\begin{tabular}{|c|c|c|c|}
\hline \multirow[t]{2}{*}{$f_{3}(x)$} & \multicolumn{3}{|c|}{ Absolute error $\widetilde{E}_{k}$ for $k=1, \ldots, 5$} \\
\hline & Chebyshev & Halley & Super-Halley \\
\hline \multirow[t]{2}{*}{$k$} & $\beta=0$ & $\beta=1 / 2$ & $\beta=1$ \\
\hline & \multicolumn{3}{|c|}{$x_{0}=0.3$ and $\alpha=0.3459548158 \ldots$} \\
\hline 1 & $5.72 \cdot 10^{-05}$ & $1.01 \cdot 10^{-04}$ & $2.79 \cdot 10^{-04}$ \\
\hline 2 & $2.95 \cdot 10^{-14}$ & $1.43 \cdot 10^{-12}$ & $5.79 \cdot 10^{-11}$ \\
\hline 3 & $4.06 \cdot 10^{-42}$ & $4.15 \cdot 10^{-36}$ & $5.16 \cdot 10^{-31}$ \\
\hline 4 & $\mathbf{1 , 0 6} \cdot 10^{-125}$ & $1.01 \cdot 10^{-106}$ & $3.66 \cdot 10^{-91}$ \\
\hline \multirow[t]{2}{*}{5} & $1.91 \cdot 10^{-376}$ & $1.45 \cdot 10^{-318}$ & $1.30 \cdot 10^{-271}$ \\
\hline & \multicolumn{3}{|c|}{$x_{0}=0.1$ and $\alpha=0.3459548158 \ldots$} \\
\hline 1 & $2.89 \cdot 10^{-02}$ & $7.21 \cdot 10^{-03}$ & $4.77 \cdot 10^{-02}$ \\
\hline 2 & $7.80 \cdot 10^{-06}$ & $5.08 \cdot 10^{-07}$ & $2.61 \cdot 10^{-04}$ \\
\hline 3 & $7.51 \cdot 10^{-17}$ & $1.85 \cdot 10^{-19}$ & $4.71 \cdot 10^{-11}$ \\
\hline 4 & $6.72 \cdot 10^{-50}$ & $8.92 \cdot 10^{-57}$ & $2.78 \cdot 10^{-31}$ \\
\hline 5 & $4.81 \cdot 10^{-149}$ & $9.99 \cdot 10^{-169}$ & $5.73 \cdot 10^{-92}$ \\
\hline$K_{3}\left(\alpha ; G_{\beta}\right)$ & -0.159 & -1.410 & -2.663 \\
\hline
\end{tabular}

Table 5: Error decreasing and asymptotic constants (best errors and the smallest asymptotic constant are indicated in boldface).

seems to converge faster. Finally, as for the basin of attraction of a method, the asymptotic constant depends on the function $f(x)$ under consideration. 


\begin{tabular}{|c||l|l|l|}
\hline \multicolumn{1}{|c||}{$f_{4}(x)$} & \multicolumn{3}{|c|}{ Absolute error $\widetilde{E}_{5}$} \\
\multicolumn{1}{|c||}{$\alpha=-3$} & Chebyshev & Halley & Super-Halley \\
& & & \\
\hline & & & $\beta=1$ \\
initial $x_{0}$ & $\beta=0$ & $\beta=1 / 2$ & \\
& & & \\
\hline-2.2 & $1.08 \cdot 10^{-15}$ & $\mathbf{3 . 1 5} \cdot 10^{-\mathbf{1 3 1}}$ & $4.28 \cdot 10^{-102}$ \\
-2.4 & $7.66 \cdot 10^{-58}$ & $\mathbf{5 . 2 8} \cdot 10^{-\mathbf{1 4 7}}$ & $1.95 \cdot 10^{-135}$ \\
-2.6 & $2.94 \cdot 10^{-112}$ & $\mathbf{4 . 1 5} \cdot 10^{-185}$ & $9.59 \cdot 10^{-184}$ \\
-2.8 & $5.03 \cdot 10^{-196}$ & $9.02 \cdot 10^{-258}$ & $\mathbf{3 . 3 1} \cdot 10^{-264}$ \\
-3.2 & $2.29 \cdot 10^{-215}$ & $6.70 \cdot 10^{-263}$ & $\mathbf{1 . 5 5} \cdot 10^{-282}$ \\
-3.4 & $3.08 \cdot 10^{-151}$ & $7.02 \cdot 10^{-194}$ & $\mathbf{4 . 2 6} \cdot 10^{-220}$ \\
-3.6 & $4.40 \cdot 10^{-117}$ & $1.03 \cdot 10^{-155}$ & $\mathbf{4 . 3 5} \cdot 10^{-189}$ \\
-3.8 & $5.61 \cdot 10^{-95}$ & $3.14 \cdot 10^{-130}$ & $\mathbf{8 . 6 6} \cdot 10^{-\mathbf{1 7 2}}$ \\
\hline \hline$K_{3}\left(\alpha ; G_{\beta}\right)$ & 0.5102 & 0.1837 & $\mathbf{- 0 . 1 4 2 9}$ \\
\hline
\end{tabular}

Table 6: Error after 5 iterations, $\widetilde{E}_{5}$, depending on the initial point $x_{0}$ (best error after 5 iterations and the best asymptotic constant are indicated in boldface). 


\begin{tabular}{|c|c|c|c|}
\hline \multirow[t]{2}{*}{$f_{5}(x)$} & \multicolumn{3}{|c|}{ Absolute error $\widetilde{E}_{k}$ for $k=1, \ldots, 5$} \\
\hline & Chebyshev & Halley & Super-Halley \\
\hline \multirow[t]{2}{*}{$k$} & $\beta=0$ & $\beta=1 / 2$ & $\beta=1$ \\
\hline & \multicolumn{3}{|c|}{$\overline{x_{0}}=0.2$ and $\alpha=0.1061045880 \ldots$} \\
\hline 1 & $4.45 \cdot 10^{-03}$ & $1.14 \cdot 10^{-02}$ & $2.08 \cdot 10^{-02}$ \\
\hline 2 & $5.46 \cdot 10^{-06}$ & $1.33 \cdot 10^{-05}$ & $4.78 \cdot 10^{-04}$ \\
\hline 3 & $1.00 \cdot 10^{-14}$ & $1.36 \cdot 10^{-14}$ & $5.47 \cdot 10^{-09}$ \\
\hline 4 & $6.19 \cdot 10^{-41}$ & $1.45 \cdot 10^{-41}$ & $8.22 \cdot 10^{-24}$ \\
\hline 5 & $1.46 \cdot 10^{-119}$ & $1.73 \cdot 10^{-122}$ & $2.79 \cdot 10^{-68}$ \\
\hline 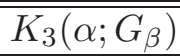 & (61.64 & 5.725 & -50.19 \\
\hline
\end{tabular}

Table 7: Error decreasing and asymptotic constants (best errors and the smallest asymptotic constant are indicated in boldface). 


\begin{tabular}{|c|c|c|c|}
\hline$F_{p}(x)$ & \multicolumn{3}{|c|}{ Absolute error $\widetilde{E}_{k}$ for $k=1, \ldots, 4$} \\
\hline$\alpha=1$ & Chebyshev & Halley & Super-Halley \\
\hline$k$ & $\beta=0$ & $\beta=1 / 2$ & $\beta=1$ \\
\hline$p=3$ & \multicolumn{3}{|c|}{$x_{0}=0.9$} \\
\hline 1 & $1.16 \cdot 10^{-03}$ & $1.06 \cdot 10^{-03}$ & $9.68 \cdot 10^{-04}$ \\
\hline 2 & $1.56 \cdot 10^{-09}$ & $1.20 \cdot 10^{-09}$ & $9.08 \cdot 10^{-10}$ \\
\hline 3 & $3.80 \cdot 10^{-27}$ & $1.71 \cdot 10^{-27}$ & $7.50 \cdot 10^{-28}$ \\
\hline 4 & $5.47 \cdot 10^{-80}$ & $4.97 \cdot 10^{-81}$ & $4.21 \cdot 10^{-82}$ \\
\hline$K_{3}\left(\alpha ; G_{\beta}\right)$ & \multicolumn{3}{|c|}{1} \\
\hline$p=5$ & \multicolumn{3}{|c|}{ 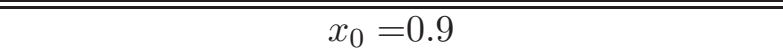 } \\
\hline 1 & $6.01 \cdot 10^{-05}$ & $6.00 \cdot 10^{-05}$ & $5.00 \cdot 10^{-05}$ \\
\hline 2 & $4.71 \cdot 10^{-21}$ & $4.67 \cdot 10^{-21}$ & $4.63 \cdot 10^{-21}$ \\
\hline 3 & $1.39 \cdot 10^{-101}$ & $1.33 \cdot 10^{-101}$ & $1.28 \cdot 10^{-101}$ \\
\hline 4 & $3.10 \cdot 10^{-504}$ & $2.52 \cdot 10^{-504}$ & $2.04 \cdot 10^{-504}$ \\
\hline$K_{5}\left(\alpha ; G_{\beta}\right)$ & \multicolumn{3}{|c|}{6} \\
\hline$p=20$ & \multicolumn{3}{|c|}{$x_{0}=0.5$} \\
\hline 1 & $1.63 \cdot 10^{-4}$ & $1.63 \cdot 10^{-04}$ & $1.63 \cdot 10^{-04}$ \\
\hline 2 & $3.02 \cdot 10^{-74}$ & $3.04 \cdot 10^{-74}$ & $3.04 \cdot 10^{-74}$ \\
\hline 3 & $6.77 \cdot 10^{-1469}$ & $7.96 \cdot 10^{-1469}$ & $9.35 \cdot 10^{-1469}$ \\
\hline 4 & $7.06 \cdot 10^{-29362}$ & $1.77 \cdot 10^{-29360}$ & $4.43 \cdot 10^{-29359}$ \\
\hline$K_{20}\left(\alpha ; G_{\beta}\right)$ & \multicolumn{3}{|c|}{171} \\
\hline
\end{tabular}

Table 8: Error decreasing and asymptotic constant for $G_{\beta}$ applied on $F_{p}$. 


\begin{tabular}{|c|c|c|c|c|}
\hline & \multicolumn{3}{|c|}{$C(\beta)=\frac{2 n-1}{3(n-1)}-\beta$} & \\
\hline & \multicolumn{3}{|c|}{ Iteration functions } & \\
\hline & Chebyshev & Halley & Super-Halley & $\begin{array}{c}\text { Optimal } \\
\text { parameter }\end{array}$ \\
\hline$n$ & $\beta=0$ & $\beta=1 / 2$ & $\beta=1$ & $\beta^{*}(n)=\frac{2 n-1}{3(n-1)}$ \\
\hline 2 & 1 & $1.5 / 3$ & 0 & $3 / 3$ \\
\hline 3 & $5 / 6$ & $2 / 6$ & $-1 / 6$ & $5 / 6$ \\
\hline 4 & $7 / 9$ & $2.5 / 9$ & $-2 / 9$ & $7 / 9$ \\
\hline 5 & $9 / 12$ & $3 / 12$ & $-3 / 12$ & $9 / 12$ \\
\hline 6 & $11 / 15$ & $3.5 / 15$ & $-4 / 15$ & $11 / 15$ \\
\hline 7 & $13 / 18$ & $4 / 18$ & $-5 / 18$ & $13 / 18$ \\
\hline 8 & $15 / 21$ & $4.5 / 21$ & $-6 / 21$ & $15 / 21$ \\
\hline 9 & $17 / 24$ & $5 / 24$ & $-7 / 24$ & $17 / 24$ \\
\hline 10 & $19 / 27$ & $5.5 / 27$ & $-8 / 27$ & $19 / 27$ \\
\hline$\vdots$ & $\vdots$ & $\vdots$ & $\vdots$ & $\vdots$ \\
\hline$\infty$ & $2 / 3$ & $1 / 6$ & $-1 / 3$ & $2 / 3$ \\
\hline
\end{tabular}

Table 9: Comparaison of the parameter $C(\beta)$ of the asymptotic constant for Chebychev, Halley and Super-Halley IFs for the $n$-th root computation problem (the smallest asymptotic constant are indicated in boldface). 


\section{Acknowledgments}

This work has been financially supported by an individual discovery grant from NSERC (Natural Sciences and Engineering Research Council of Canada).

\section{References}

[1] I.K. Argyros, F. Szidarovszky, The Theory and Applications of Iteration Methods, CRC Press, USA (1993).

[2] M. Basto, V. Semiao, F.L. Calheiros, A new iterative method to compute nonlinear equations, Appl. Math. Comput., 173, No. 1 (2006), 468-483, doi: j.amc.2005.04.045.

[3] E. Bodewig, Sur la méthode Laguerre pour l'approximation des racines de certaines équations algébriques et sur la critique d'Hermite, Indag. Math., 8 (1946), 570-580.

[4] F. Dubeau, C. Gnang, On the Chebyshev-Halley family of iteration functions and the n-th root computation problem, Int. J. Pure Appl. Math., 87, No. 3 (2013), To Appear.

[5] W. Gander, On Halley's iteration method, Amer. Math. Month., 92, No. 2 (1985), 131-134, doi: 10.2307/2322644.

[6] J. Gerlach, Accelerated convergence in Newton's method, SIAM Review, 36, No. 2 (1994), 272-276, doi: 10.1137/1036057.

[7] J.M. Gutiérrez, M.A. Hernandez, A family of Chebyshev-Halley type methods in Banach spaces, Bull. Austral. Math. Soc., 55, No. 1 (1997), 113-130, doi: $10.1017 /$ S0004972700030586.

[8] J.M. Gutiérrez, M.A. Hernández, An accaleration of Newton's method: Super-Halley method, Appl. Math. Comput., 117. No. 2-3 (2001), 223-239, doi: 10.1016/S0096-3003(99)00175-7

[9] J.M. Gutiérrez, M.A. Hernández, M.-A. Salanova, Calculus of $n$th roots and third order iterative methods, Nonlinear analysis, 47, No. 4 (2001), 2875-2880, doi: 10.1016/S0362-546X(01)00408-4.

[10] B. Neta, M. Scott, C. Chun, Basins of attraction for several methods to find simple roots of nonlinear equations, Appl. Math. Comput., 218, No. 21 (2012), 10548-10556, doi: 10.1016/j.amc.2012.04.017. 
[11] M.S. Petković, Comments on the Basto-Semiao-Calheiros root finding method, Appl. Math. Comput., 184, No. 2 (2007), 143-148, doi: 10.1016/j.amc.2006.05.201.

[12] J.F. Traub, Iterative Methods for the Solution of Equations, Prentice-Hall, USA (1964), doi: 10.2307/2004117.

[13] W. Werner, Some improvements of classical iterative methods for the solution of nonlinear equations, In: Numerical Soution of Nonlinear equations, (Proc., Bremen, 1980), E.L. Allgower, K. Glashoff and H.O. Peitgen, eds, Lecture Notes in Math., 878, (1981), 427-440, doi: 10.1007/BFb0090691. 
